\title{
What are some of the key inputs that we as the dental profession (Dentists, Therapists, Hygienists, Technicians, Specialist and Assistants) should agree upon and aim to achieve under the $\mathrm{NHI}$ ?
}

- an opinion piece submitted to the SADJ

SADJ November 2019, Vol. 74 No. 10 p530 - p531

\section{J Mthethwa}

Dear Editor,

I am aware that the NHI Bill is out there with a deadline for comments by the end of November. I have decided to pen down some of my personal views and opinions on how we as the profession should go about implementing the $\mathrm{NHI}$ in this country.

These are my personal views as informed by the many years I spent in private practice as a dentist both in this country and abroad, my years as a Senior Government Official in the Province, my interaction with colleagues formally and informally and lastly my interaction with academics and students who are concerned about the future of the profession. I therefore write here in my personal capacity having perused through the summary of the Bill.

Below are some of the key principles on working arrangements on which we, as the profession, need to agree. We need to ensure that in the $\mathrm{NHI}$ environment:

- We promote a strong multidisciplinary approach. This implies that we need dentist-led teams with dental therapist, oral hygienist, dental technician and dental assistants working side by side, each freely exercising his/her scope of practice.

- We promote group practice models and discourage solo practice models.

- There are various contracting models for primary and secondary care in general dental practice and tertiary/quaternary services through Hospital care.

\section{Author affiliation:}

Jimmy Mthethwa: BDS (Medunsa), PG Cert (UK), MPH (UK), MBA

(UK), Stomatologist, Department of Oral Hygiene, Community Den-

tistry, School of Oral Health Sciences, University of Witwatersrand Johannesburg, South Africa.

Email: jimmy.mthethwa@wits.ac.za

ORCID Number: 0000-0002-3586-1930

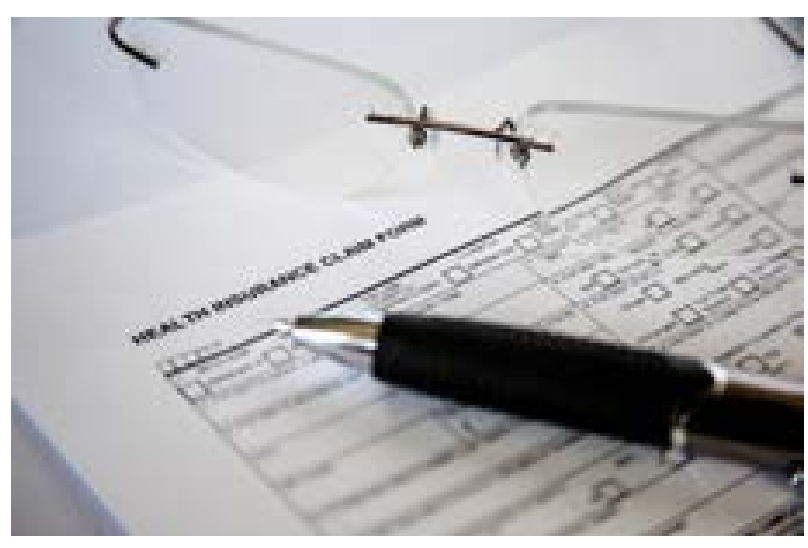

- Acceptable and cost-effective clinical protocols and guidelines to be used to guide clinicians are developed by the profession.

- There is a proper referral system to guide the profession and to be followed by patients.

- There are local contracting mechanisms of dental services within district health authority, moving away from central government.

- Dental service packages are developed for all population groups, serving children, adults, elderly, special needs patients and for prison settings.

On the key Ministerial Committees, we need to ensure that at least one or two members of the dental profession are included in each of the key committees such as:

- Benefits Advisory Committee,

- Health Benefits Pricing

- Stakeholder Advisory Committees

- Technical Committee including

- Remuneration Committees 
On the Benefits Advisory Committee, we need to ensure that:

- There is a recognition that Oral Health is not just Primary Health care alone.

- Oral Health benefits under NHI must be defined across all levels of health care from Primary to Secondary, Tertiary and to Quaternary services.

- The design of package of services will be executed by the Oral Health Profession.

- There are norms, standards and prescribed minimum benefits for Oral Health.

- Clinical protocols and guidelines for Oral Health are developed in this committee

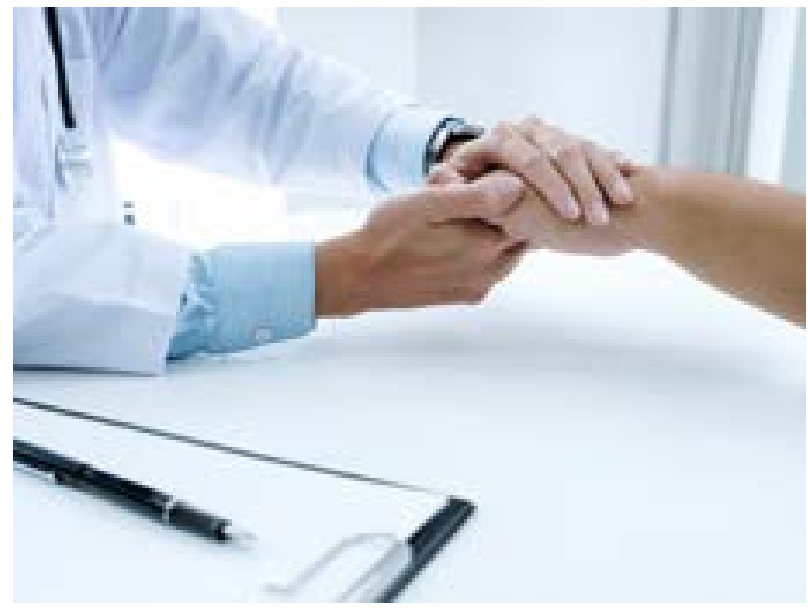

On dental Information systems, we need to ensure that:

- A universal standardized electronic clinical record system for Oral Health should be developed.

- Such a system can interface with other data bases such as the medical fraternity, Home Affairs, etc.

- Minimum data indicators are developed for Oral Health at various levels of care.

- The system should include radiology, pharmacology, laboratory and clinical record modules.

- The system must be able to interface at all levels of care, primary through to quaternary care.

- The system must be compatible with other systems and be upgradable to ensure future sustainability.

On the role of central hospitals and academic Oral Health Centres, we need to consider that:

- There are four Academic Oral Health centres in the country.

- These are classified as central/quaternary services.

- These must be under the National Department of Health and be centrally managed as semi-autonomous centres focusing on teaching and research, with service delivery on tertiary and quaternary levels only. Each centre should be allowed to raise and retain its additional revenue.

- These four centres must support the nine provinces in terms of training, research etc.

- The country needs to invest in the KZN/UKZN Dental Therapy and Oral Hygiene School, developing it into a full Dental School offering all other disciplines and training.
There is a need to consider the possibility of establishing an additional Dental School elsewhere in the country as driven by need and evidence.

These training platforms must focus on primary prevention and develop middle level professional development over and above the tertiary and quaternary focus.

Finally, there are some grey areas that I think need more attention and consensus from amongst the professions:

- The Bill needs to be clear on the eligibility of refugees and foreign nationals for Oral Health services as the current model may be unsustainable.

- The Bill needs to define Oral Health services which may be excluded from the $\mathrm{NHI}$ and may then be channeled to private medical insurance.

- The Office of Health Standards and Compliance needs to strengthen the Inspectorate for Oral Health in the inspection of dental practices and dental facilities in the hospitals to ensure appropriate equipment and settings are available.

- The Capitation vs. the Fee for Service models of reimbursement need to be balanced in a hybrid system to counter the effects of both under- and over-serving of patients.

\section{In conclusion:}

Clearly there is a lot that the profession needs to work on and develop rapidly in the coming weeks, months and years. The profession needs to put differences aside, for it can ill afford fragmentation and fighting over small pieces of the pie. We must work together as one united Oral Health Profession voice.

The NHI Bill provides the profession with an opportunity to start afresh, to put its house in order and to fix Oral Health not just for now but for the benefit of future generations to come. If all these objectives are achieved it would be easier to ensure that Government listens to one voice of Oral Health and to ensure that Oral Health is an integral part of the General Health service.

Yours faithfully,

\section{Dr Jimmy Mthethwa}

